\title{
Dynamic Interaction among the Platform Domain and Two Membrane- Proximal Immunoglobulin-Like Domains of Class I Major Histocompatibility Complex: Normal Mode Analysis
}

\author{
Hiroyuki NoJima, ${ }^{a, \#}$ Mayuko TAKedA-Shitaka, ${ }^{a, \#}$ Kazuhiko Kanou, ${ }^{a}$ Kenshu KamiYa, ${ }^{b}$ and \\ Hideaki UMEYAMA *,a \\ ${ }^{a}$ School of Pharmaceutical Sciences, Kitasato University; 5-9-1 Shirokane, Minato-ku, Tokyo 108-8641, Japan: and \\ ${ }^{b}$ School of Science, Kitasato University; 1-15-1 Sagamihara, Kanagawa 228-8555, Japan. \\ Received November 4, 2007; accepted February 27, 2008; published online March 4, 2008
}

\begin{abstract}
Class I major histocompatibility complex (MHC) molecules have three domains, a platform domain and two membrane-proximal immunoglobulin-like domains, an $\alpha 3$ domain and a $\beta_{2}$-immunoglobulin $\left(\beta_{2} \mathrm{~m}\right)$. To understand the dynamic interactions among the three domains, we simulated the behavior of a partial model deficient in $\beta_{2} \mathrm{~m}$ and another model deficient in the $\alpha 3$ domain, by normal mode analysis. As a result, the partial model deficient in $\beta_{2} \mathrm{~m}$ was more flexible in interdomain conformation than the other model. The lowest frequency modes $\left(<2 \mathrm{~cm}^{-1}\right)$ observed for the simulations of the partial model deficient in $\beta_{2} \mathrm{~m}$ showed clear interdomain motions as if each domain moved like a rigid body. Such low frequencies and clear interdomain motions were not observed for the simulations of the other model, therefore the interdomain flexibility of the partial model deficient in $\beta_{2} \mathrm{~m}$ may be due to the lowest frequency modes $\left(<2 \mathrm{~cm}^{-1}\right)$. These results suggest that $\beta_{2} \mathrm{~m}$ contributes to maintaining the interdomain conformation of class I MHC molecules more than the $\alpha 3$ domain does, and may offer convincing evidence to support the notion that the $\alpha 3$ domain and $\beta_{2} \mathrm{~m}$ do not have an equal influence on the structural stability of class I MHC molecules.
\end{abstract}

Key words class I major histocompatibility complex; $\beta_{2}$-immunoglobulin; $\alpha 3$ domain; membrane-proximal immunoglobulinlike domain; platform domain; normal mode analysis

Class I major histocompatibility complex (MHC) molecules play an important role in the immune response against infections with intracellular pathogens. During the immune response, class I MHC molecules bind to various antigen peptides (9-10 residues) in the endoplasmic reticulum (ER), and present the peptides on the cell surface to cytolytic $\left(\mathrm{CD}^{+}\right) \mathrm{T}$ cells

The structural details of class I MHC molecules with a peptide have been investigated by X-ray crystallography of many class I MHC-peptide complexes. ${ }^{1)}$ Class I MHC molecules are composed of a heavy chain and a $\beta_{2}$-immunoglobulin $\left(\beta_{2} \mathrm{~m}\right)$. The heavy chain is divided into two domains, a platform domain ( $\alpha 1$ and $\alpha 2$ domains) and an immunoglobulin-like $\alpha 3$ domain. The platform domain is composed of two $\alpha$-helical regions and an eight-stranded $\beta$-sheet, and the bound peptide is accommodated between the two $\alpha$-helical regions on the $\beta$-sheet. The $\alpha 3$ domain and $\beta_{2} \mathrm{~m}$ are located between the platform domain and the cell surface; therefore, they are called membrane-proximal immunoglobulin-like domains (Fig. 1). The two membrane-proximal immunoglobulin-like domains influence the activity and structural stability of class I MHC molecules. For example, endogenous $\beta_{2} \mathrm{~m}$ in class I MHC molecules is easily exchanged for exogenous $\beta_{2} \mathrm{~m}$ at the cell surface, ${ }^{2-7)}$ but the molecules deficient in $\beta_{2} \mathrm{~m}$ (the heavy chain and a bound peptide) have poor folding stability, and have never been crystallized. On the other hand, the molecules deficient in the $\alpha 3$ domain have been crystallized and their structure is similar to the corresponding part of the intact structure including the $\alpha 3$ domain. ${ }^{8)}$ These results suggest that the $\alpha 3$ domain and $\beta_{2} \mathrm{~m}$ do not exert an equal influence on the structural stability of class I MHC molecules.

As a complementary tool to experimental methods, mo- lecular dynamics simulations have been used to study MHC-peptide complexes. These simulations have neglected the influence of the two membrane-proximal immunoglobulin-like domains. ${ }^{9-16)}$ In recent years, the influence of the two domains has been simulated by molecular dynamics. ${ }^{17)}$ The simulations, however, have been performed for two models, a whole model composed of all domains and a partial model composed of only the platform domain, and the discussion has only focused on the conformation of the platform domain. We gave attention to the dynamic interactions among the platform domain, the $\alpha 3$ domain and $\beta_{2} \mathrm{~m}$, and then simulated the behavior of a partial model deficient in the

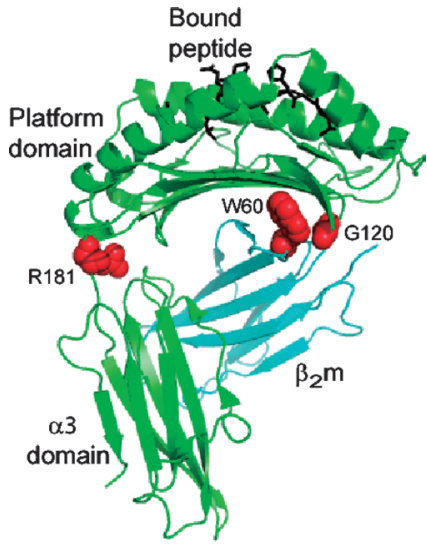

Fig. 1. Schematic Structures of HLA-A2 Class I MHC Molecules (PDB Code, 1HHJ)

The platform and $\alpha 3$ domains are represented in green. $\beta_{2} \mathrm{~m}$ is represented in blue The bound peptide is represented by black line. R181 in the heavy chain (red balls) is located at the linker position between the platform and $\alpha 3$ domains. G120 in the heavy chain (red balls) and W60 in $\beta_{2} \mathrm{~m}$ (red balls) are closest to each other in the platform domain and $\beta_{2} \mathrm{~m}$. 
$\alpha 3$ domain and another model deficient in $\beta_{2} \mathrm{~m}$, by normal mode analysis. Molecular dynamics simulation of molecules of similar size to class I MHC molecules can be used to observe vibrational modes up to $10-100 \mathrm{~ns}$, with currently available computational capability. However, normal mode analysis has the advantage of low computational costs, theoretical completeness, and clearness of harmonic vibrational modes that express the collective motions of large molecules. ${ }^{18-25)}$

\section{Experimental}

Methods. Models Used for Normal Mode Analysis The initial atomic coordinates for normal mode analysis were taken from the X-ray crystallographic structure of HLA-A2 class I MHC molecules with a bound peptide derived from HIV reverse transcriptase, ILKEPVHGV (PDB code, $\left.1 \mathrm{HHJ}^{1)}\right)$. In this PDB, the atomic coordinates of an entire complex composed of an A-chain of 275 residues (G1-E275, heavy chain,) a B-chain of 100 residues $\left(\mathrm{M} 0-\mathrm{M} 99, \beta_{2} \mathrm{~m}\right.$ ) and a C-chain of nine residues (ILKEPVHGV, bound peptide) was used as the "whole model," that is, as the standard model to compare the later two models. The atomic coordinates in which the $\alpha 3$ domain (T182-E275) was removed from the "whole model" were used as the " $\alpha 3$ domain-removed model." On the other hand, the atomic coordinates in which $\beta_{2} \mathrm{~m}$ was removed from the "whole model" were used as the " $\beta_{2}$ m-removed model."

Normal Mode Analysis To obtain the structure for performing normal mode analysis, energy optimization from the initial coordinates was performed for each model. In energy optimization, bond length, bond angle and dihedral angle are treated as parameters. ${ }^{26)}$ A "slightly modified" Assisted Model Building with Energy Refinement (AMBER) united atom-force field was used for energy optimization. ${ }^{27,28)}$ "Slightly modified" means that a "suspected potential," in which the disulfide bond is only treated as a function of the distance between $\mathrm{S}$ atoms, is introduced instead of the bond-angle and dihedral-angle parameters of the disulfide bond. ${ }^{26)}$ To consider short-distance electrostatic interaction, a distance-dependent dielectric constant $(r / \AA)$ for electrostatic energy was used. ${ }^{23,29)}$ Position-restriction energy, E, expressed in Eq. 1 was imposed in energy optimization process.

$$
E=\sum_{i}^{N} K\left(r_{i}-r_{i}^{0}\right)^{2}
$$

In Eq. $1, K(\mathrm{kcal} / \mathrm{mol} \AA)$ is a force constant, and $r_{i}$ and $r_{i}^{0}$ are the displaced and initial coordinates of the $i$ th atom, respectively. The above restriction was gradually relaxed by decreasing the $K$ value so that normal mode analysis could be executed near the initial coordinates. A threshold of
$0.04 \mathrm{kcal} / \mathrm{mol} \AA$ for the maximal component of the gradients of atoms was used for energy optimization. To avoid accidental errors when comparing between models, six energy-optimized structures for the normal mode simulations were obtained for a model, by using six different relaxation patterns. ${ }^{30-33)}$

Normal mode analysis was performed using programs developed by our laboratory. ${ }^{26)}$ In the calculation, only dihedral angles were treated as a parameter. RMS fluctuation of the $i$ th $\mathrm{C} \alpha$ atom, $F_{i}$, was calculated by the following equation ${ }^{18)}$ :

$$
\left\langle F_{i}^{2}\right\rangle=\left\langle\Delta r_{i}^{2}\right\rangle=k_{\mathrm{B}} T \sum_{k}\left(\left|\mathbf{a}_{k l}\right|^{2} / \omega_{k}^{2}\right)
$$

in which $\mathbf{a}_{k i}$ is the vector of projection of the $k$ th normal mode with frequency $\omega_{k}$ on the Cartesian components of the displacement vector $\Delta \mathbf{r}_{i}, k_{\mathrm{B}}$ is the Bolzmann constant, and $T$ is the absolute temperature. Motional correlation coefficient between the $i$ th and $j$ th $\mathrm{C} \alpha$ atoms, $D_{i j}$, was calculated by the following equation ${ }^{20)}$ :

$$
\left\langle D_{i j}^{2}\right\rangle=\left\langle\Delta r_{i} \cdot \Delta r_{j}\right\rangle /\left\{\left\langle\Delta r_{i}^{2}\right\rangle^{1 / 2}\left\langle\Delta r_{j}^{2}\right\rangle^{1 / 2}\right\}
$$

$\mathrm{C} \alpha$ atom RMS fluctuations and motional correlation coefficients between $\mathrm{C} \alpha$ atoms were calculated using our program, assuming a temperature of $300 \mathrm{~K}^{30-36)}$ Each value of the fluctuations and the motional correlation coefficients for a model was determined by averaging the six values obtained from the simulations of the six energy-optimized structures of the model.

To lower computational cost, we adopted AMBER united atom-force field in which aliphatic and aromatic carbon-bonded hydrogen atoms were neglected but introduced three kinds of suspected aliphatic and aromatic carbon atoms whose atomic weights were $13.02,14.03$ or 15.03. Higo and Umeyama have reported that even the backbone model where only $\mathrm{C} \alpha$ atoms were explicitly treated neglecting the other atoms is useful in obtaining collective motions of a protein without heavy simulations by assigning appropriate pairwise interactions between $\mathrm{C} \alpha$ atoms. ${ }^{37)}$ Therefore, only the introduction of the above suspected carbon atoms may have little influence upon the essential characteristic of collective motions of a protein. Indeed, $\mathrm{C} \alpha$ atom RMS fluctuations expressed by the whole motion of the "whole model" showed good correlation in pattern with the X-ray B-factors of the corresponding structure (Fig. 2). Consequently, we confirmed that introduction of this force field was not irrelevant.

Generally, the dynamic characteristics of large molecules are heavily influenced by low frequency modes. ${ }^{38)}$ In this study, $\mathrm{C} \alpha$ atom RMS fluctuations and motional correlation coefficients between $\mathrm{C} \alpha$ atoms were expressed in low frequency modes $\left(<50 \mathrm{~cm}^{-1}\right)$, because the fluctuation patterns and the correlation map profiles expressed in low frequency modes were little different from those expressed in all-frequency modes (data not shown). Significant differences by subtraction of the fluctuations between

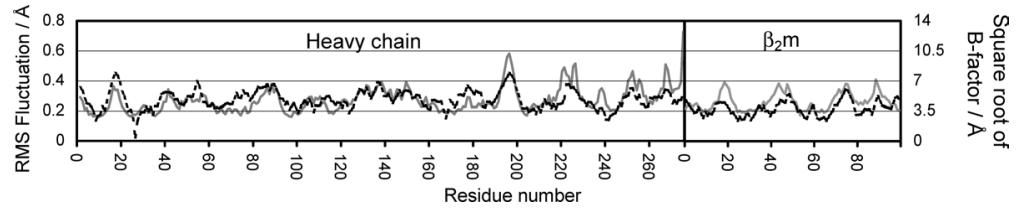

Fig. 2. Comparison between X-Ray B-Factors (Broken Black Line, Refer to the Right Vertical Axis) and C $\alpha$ Atom RMS Fluctuations (Gray Line, Refer to the Left Vertical Axis)

\begin{tabular}{|c|c|c|c|c|}
\hline & & "Whole model" & " $\alpha 3$ domain-removed model" & " $\beta_{2} \mathrm{~m}$-removed model" \\
\hline \multirow[t]{4}{*}{ Number of residues } & Total & 384 & 290 & 281 \\
\hline & Heavy chain & $275(\mathrm{G} 1-\mathrm{E} 275)$ & $181(\mathrm{G} 1-\mathrm{R} 181)$ & $275(\mathrm{G} 1-\mathrm{E} 275)$ \\
\hline & $\beta_{2} \mathrm{~m}$ & 100 (M0-M99) & 100 (M0-M99) & - \\
\hline & Bound peptide & $9(\mathrm{I} 1-\mathrm{V} 9)$ & $9(\mathrm{I} 1-\mathrm{V} 9)$ & $9(\mathrm{I} 1-\mathrm{V} 9)$ \\
\hline RMS deviations $^{a)}$ & & $1.75-2.03$ & $1.42-1.45$ & $1.73-2.02$ \\
\hline Number of modes & $\begin{array}{l}\text { Total } \\
<50 \mathrm{~cm}^{-1 b)}\end{array}$ & $\begin{array}{c}1674 \\
293-303\end{array}$ & $\begin{array}{c}1289 \\
223-226\end{array}$ & $\begin{array}{c}1224 \\
222-226\end{array}$ \\
\hline
\end{tabular}

The fluctuations were expressed by the whole motion of the "whole model."

Table 1. Summary of Three Models Used for Energy Optimization and Normal Mode Analysis

a) RMS deviations were calculated between an original model and the six energy-optimized structures derived from the original model. $\quad b$ ) Number of modes $<50 \mathrm{~cm}^{-1}$ have a range because the values of normal mode calculations are slightly different between the six energy-optimized structures. 
two models, (significance level 2.5\%) were estimated by Wilcoxon's rank sum test, a nonparametric test. ${ }^{30-33)}$ When attention is focused on the motion of a component, it can be broken down into internal and external motions. $^{23)}$ To compare with the whole motion of the " $\alpha 3$ domain-removed model," the internal motion of the "platform domain and $\beta_{2}$ m" component in the "whole model" were extracted by Eckart's condition. ${ }^{39)}$ To compare with the whole motion of the " $\beta_{2}$ m-removed model," the internal motion of the "heavy chain" component in the "whole model" was extracted by the same condition. To observe the internal motion of a domain, Eckart's condition was also applied to each domain. The summary of the three models used for energy optimization and normal mode analysis are shown in Table 1.

\section{Results}

$\mathrm{C} \alpha$ Atom RMS Fluctuations Plotted in Order of Residue Number $\mathrm{C} \alpha$ atom RMS fluctuations for the three models were plotted in order of residue number (Fig. 3). The fluctuations expressed by the whole motion of the " $\alpha 3$ domain-removed model" were superimposed on those extracted from the internal motion of the "platform domain and $\beta_{2} \mathrm{~m}$ " component in the "whole model" (Fig. 3A). The " $\alpha 3$ domain-removed model" showed fluctuation differences with a peak (significant difference $>0.05 \AA$ ) at nine points $(1-4$, $28-32,49-50,102-111$ and $169-181$ in the platform domain, and $7-26,41-52,66-79$ and $92-99$ in $\beta_{2} \mathrm{~m}$ ) compared with the "whole model" (black line in Fig. 3A). These peaks clustered on the loops or the terminals, that is, the outside of the molecules. The fluctuations expressed by the whole motion of the " $\beta_{2}$ m-removed model" were superimposed on those extracted from the internal motion of the "heavy chain" component in the "whole model" (Fig. 3B). The " $\beta_{2}$ m-removed model" showed fluctuation differences at all $\mathrm{C} \alpha$ atoms compared with the "whole model," and the fluctuation differences were very large compared with those of the " $\alpha 3$ domain-removed model" versus the "whole model" (black line in Fig. 3B).

To examine how significantly the fluctuation differences shown in Figs. 3A and B reflected the internal motion of a domain, the fluctuations were extracted from the internal motion of each domain (Figs. 3C, D). The " $\alpha 3$ domain-removed model" showed fluctuation differences with a peak (significant difference $>0.05 \AA)$ at four points $(105-106$ in the platform domain, and $12-14,57-58$ and $95-99$ in $\beta_{2} \mathrm{~m}$ ) compared with the "whole model" (black line in Fig. $3 \mathrm{C}$ ). On the other hand, the " $\beta_{2} \mathrm{~m}$ domain-removed model" showed definite fluctuation differences with a peak (significant difference $>0.05 \AA)$ at seven points $(10-11,15-18$, $104-106,119-122$ and $180-181$ in the platform domain,
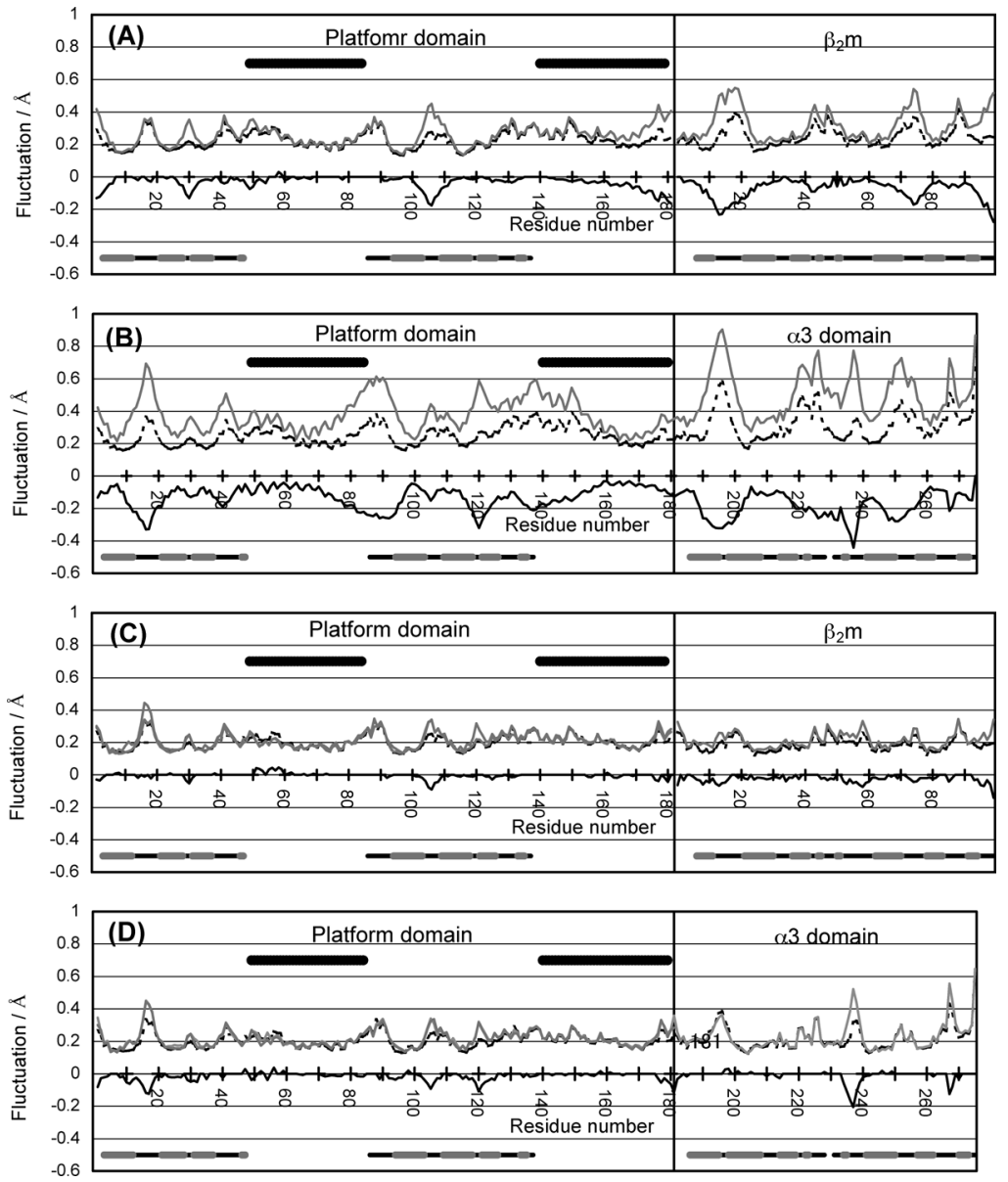

Fig. 3. $\mathrm{C} \alpha$ Atom RMS Fluctuations Plotted in Order of Residue Number

In each figure, the residues forming $\alpha$-helical regions, $\beta$-strands and loops are shown by black bars at the top, and thick gray bars and thin black bars at the bottom, respectively. (A) Fluctuations extracted from the internal motion of the "platform domain and $\beta_{2}$ m" component in the "whole model" (broken black line) and expressed by the whole motion of the " $\alpha 3$ domain-removed model" (gray line). (B) Fluctuations extracted from the internal motion of the "heavy chain" component in the "whole model" (broken black line) and expressed by the whole motion of the " $\beta_{2}$ m-removed model" (gray line). (C) Fluctuations extracted from the internal motion of each domain in the "whole model" (broken black line), and extracted from the internal motion of each domain in the " $\alpha 3$ domain-removed model" (gray line). (D) Fluctuations extracted from the internal motion of each domain in the "whole model" (broken black line), and extracted from the internal motion of each domain in the " $\beta_{2}$ m-removed model." In each figure, significant differences between the two models are shown by black lines (Wilcoxon's non-parametric test). 
and $234-239$ and $267-268$ in the $\alpha 3$ domain) compared with the "whole model" (black line in Fig. 3D). The fluctuation differences shown in Figs. 3C and D, however, were very trivial compared with those shown in Figs. 3A and B, respectively. These results indicate that the fluctuation differences shown in Figs. 3A and B hardly reflect the internal motion of each domain but the relative motion between domains, that is, the interdomain motion.

$\mathrm{C} \alpha$ Atom RMS Fluctuations Plotted in Order of Distance from a Specific $\mathbf{C} \boldsymbol{\alpha}$ Atom If the behavior of a molecule is reflected by the interdomain motion, each domain in the molecule has a tendency to move like a rigid body around the center of molecule; as a result, plotting the fluctuations of the domain in order of distance from the position may describe the fluctuation increases with the distance. Thus, $\mathrm{C} \alpha$ atom RMS fluctuations for each domain were plotted in order of distance from a specific $\mathrm{C} \alpha$ atom (Fig. 4). When the fluctuations of the " $\alpha 3$ domain-removed model" were plotted, the horizontal axes were arranged in order of distance from the $\mathrm{C} \alpha$ atom of G120 for the platform domain and the $\mathrm{C} \alpha$ atom of W60 for $\beta_{2} \mathrm{~m}$ (Fig. 4A). The two residues were chosen because they were closest to each other in the platform domain and $\beta_{2} \mathrm{~m}$. On the other hand, when the fluctuations of the " $\beta_{2}$ m-removed model" were plotted, the horizontal axes were arranged in order of distance from the $\mathrm{C} \alpha$ atom of R181, which was located at the linker position between the platform and $\alpha 3$ domains (Fig. 4B).

The arranged fluctuations expressed by the whole motion of the " $\alpha 3$ domain-removed model" were superimposed on those extracted from the internal motion of the "platform domain and $\beta_{2} \mathrm{~m}$ " component in the "whole model" (Fig. 4C). The fluctuation differences of the " $\alpha 3$ domain-removed model" versus the "whole model" were limited at both edges, the far side from G120 (>30 $\AA$ ) in the platform domain and the far side from W60 $(>20 \AA)$ in $\beta_{2} \mathrm{~m}$, and were not observed at the contact region between the platform domain and $\beta_{2} \mathrm{~m}$ (black line in Fig. 4C). When the arranged fluctuations expressed by the whole motion of the " $\beta_{2}$ m-removed model" were superimposed on those extracted from the internal motion of the "heavy chain" component in the "whole model", the fluctuation differences of the " $\beta_{2}$ m-removed model" versus the "whole model" were spread throughout the whole of the molecule and increased with distance from the linker position (black line in Fig. 4D).

The fluctuations extracted from the internal motion of a domain were also plotted in order of distance from a specific $\mathrm{C} \alpha$ atom for each domain (Figs. 4E and F). As a result, the fluctuation differences did not increased with distance in the two comparisons, the " $\alpha 3$ domain-removed model" versus the "whole model" and the " $\beta_{2}$ m-removed model" versus the "whole model" (black lines in Figs. 4E and F).

Motional Correlation Maps between $\mathbf{C} \boldsymbol{\alpha}$ Atoms To observe motional direction of the three models, motional correlation coefficients between $\mathrm{C} \alpha$ atoms were described according to distance-proportional plotting, in the same manner as described in the previous paragraph (Fig. 5). When the motional correlation map expressed by the whole motion of the " $\alpha 3$ domain-removed model" (Fig. 5B) was compared with that extracted from the "platform domain and $\beta_{2} \mathrm{~m}$ " component of the "whole model" (Fig. 5A), the " $\alpha 3$ domainremoved model" showed definite negative correlations (mo-
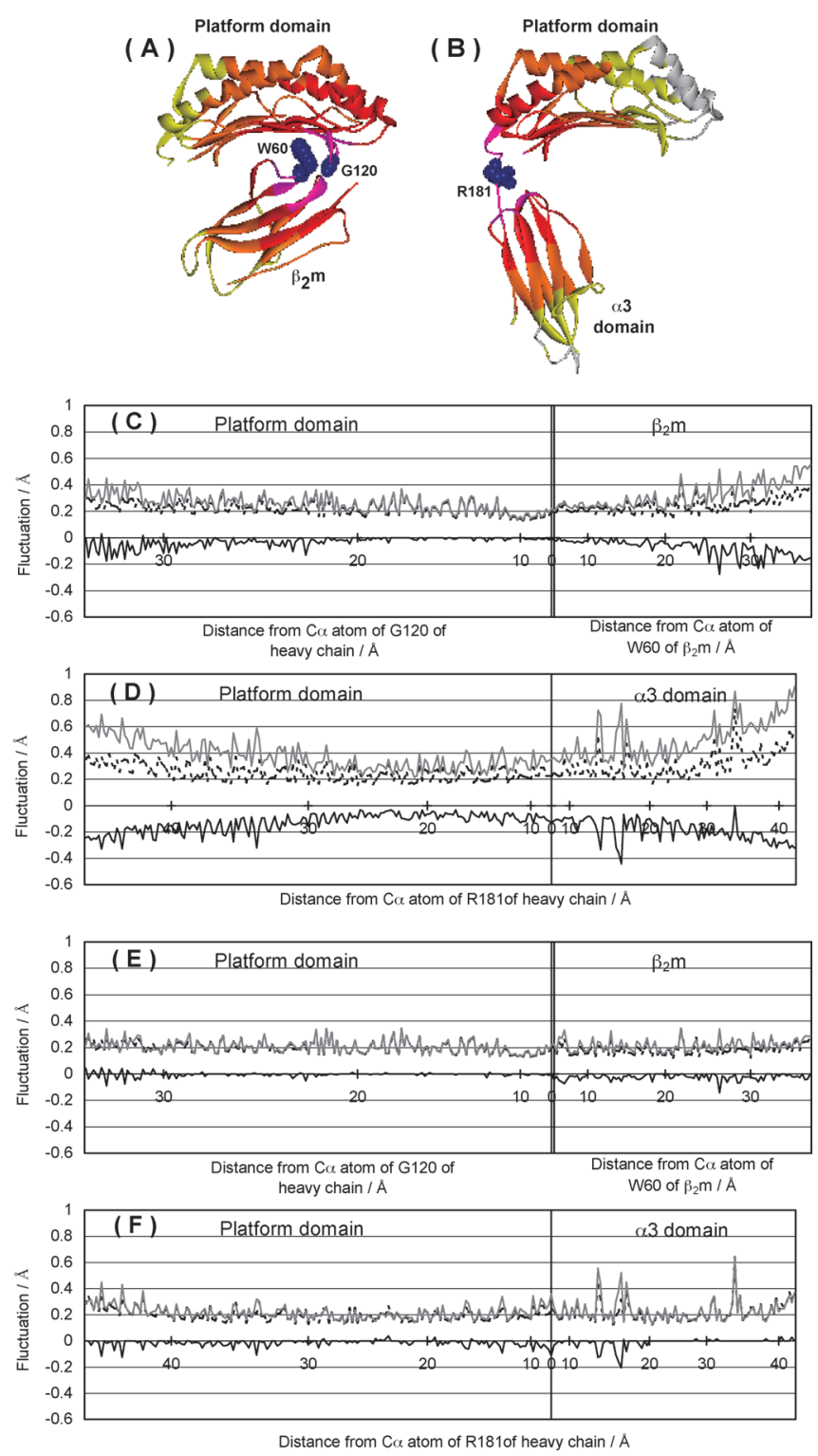

Fig. 4. $\mathrm{C} \alpha$ Atom RMS Fluctuations Plotted in Order of Distance from a Specific $\mathrm{C} \alpha$ Atom

(A) Distance-gradation map of the " $\alpha 3$ domain-removed model." The platform domain and $\beta_{2} \mathrm{~m}$ are colored in order of distance from the C $\alpha$ atom of G120 (blue balls) in the platform domain and distance from the $\mathrm{C} \alpha$ atom of W60 (blue balls) in $\beta_{2} \mathrm{~m}$, respectively. (B) Distance-gradation map of the " $\beta_{2}$ m-removed model." The platform and $\alpha 3$ domains are colored in order of distance from the $\mathrm{C} \alpha$ atom of R181 (blue balls). In (A) and (B), the residue whose distance from the standard $\mathrm{C} \alpha$ atom is less than 10, 20, 30,40 and $50 \AA$ is colored with purple, red, orange, yellow and white, respectively. (C) Fluctuations extracted from the internal motion of the "platform domain and $\beta_{2}$ m" component in the "whole model" (broken black line), and expressed by the whole motion of the " $\alpha 3$ domain-removed model" (gray line). (D) Fluctuations extracted from the internal motion of the "heavy chain" component in the "whole model" (broken black line), and expressed by the whole motion of the " $\beta_{2}$ m-removed model" (gray line). (E) Fluctuation extracted from the internal motion of each domain in the "whole model" (broken black line), and extracted from the internal motion of each domain in the " $\alpha 3$ domain-removed model" (gray line). (F) Fluctuations extracted from the internal motion of each domain in the "whole model" (broken black line), and extracted from the internal motion of each domain in the " $\beta_{2}$ m-removed model" (gray line). In (C) and (E), the $\mathrm{C} \alpha$ atoms in the platform domain and $\beta_{2} \mathrm{~m}$ are arranged in order of distance from the $\mathrm{C} \alpha$ atom of G120 in the platform domain and from the $\mathrm{C} \alpha$ atom of W60 in $\beta_{2} \mathrm{~m}$, respectively. In (D) and (F), the $\mathrm{C} \alpha$ atoms in the platform and $\alpha 3$ domains are arranged in order of distance from the $\mathrm{C} \alpha$ atom of $\mathrm{R} 181$. In $(\mathrm{C}-\mathrm{F})$, significant differences between the two models are shown by black lines (Wilcoxon's non-parametric test)

tional correlation coefficient $<-0.4)$ in a limited region, be-

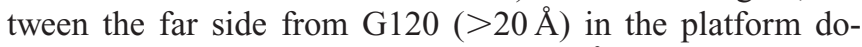
main and the far side from W60 $(>20 \AA)$ in $\beta_{2} \mathrm{~m}$. On the 


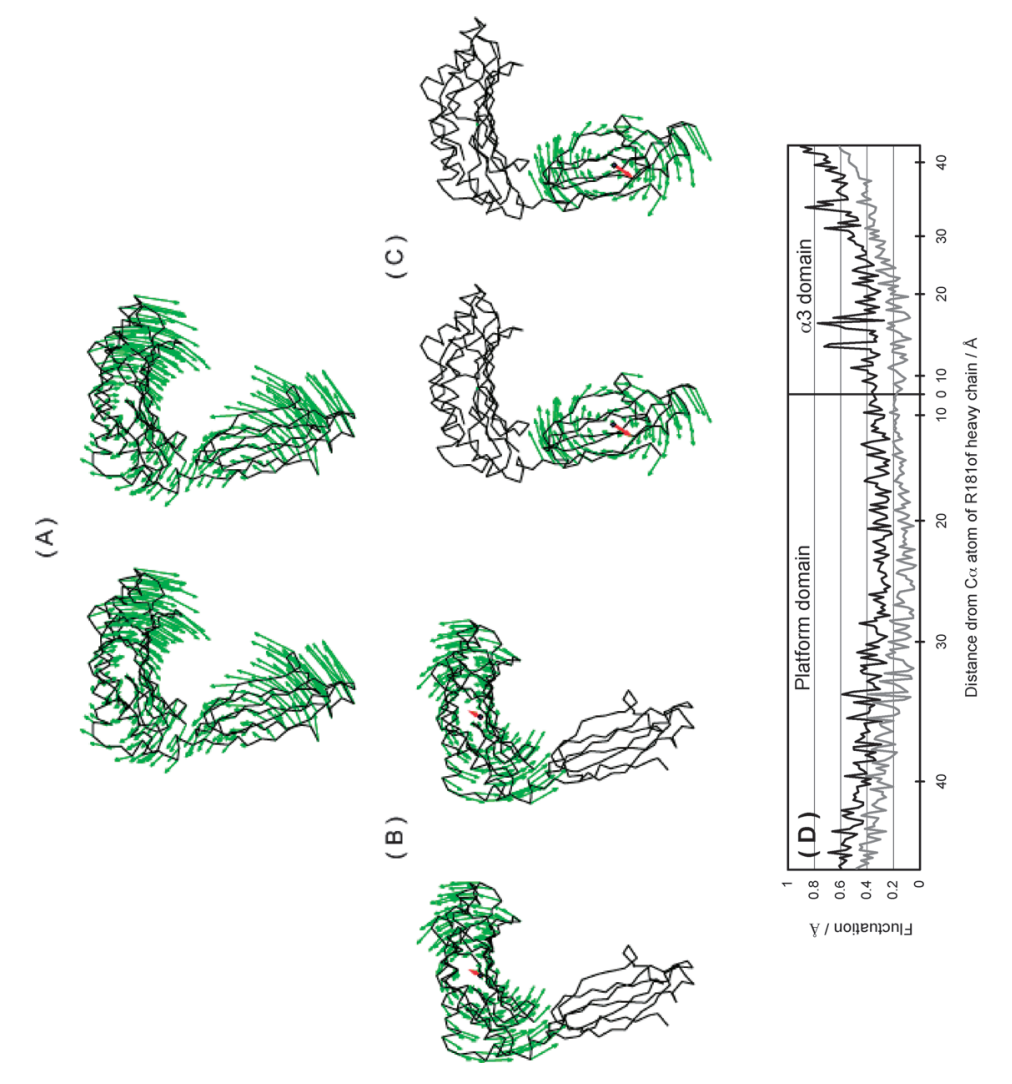

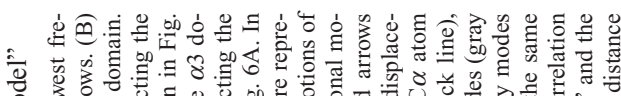

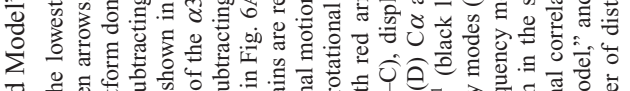

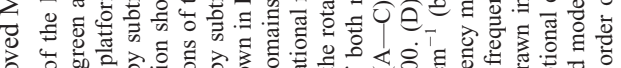

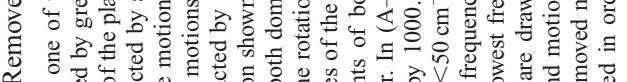

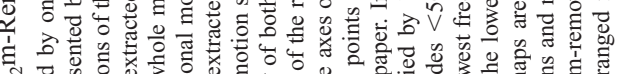

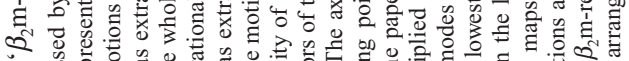

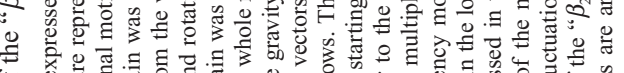

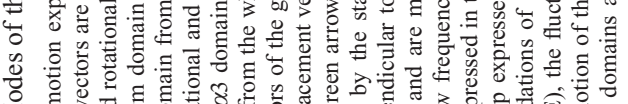

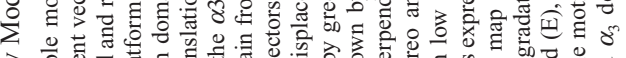

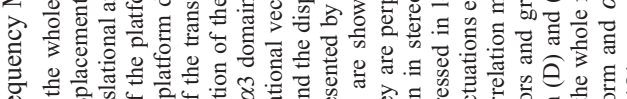

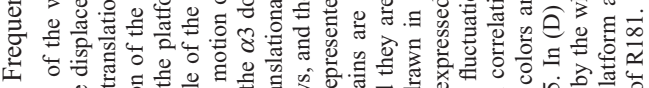

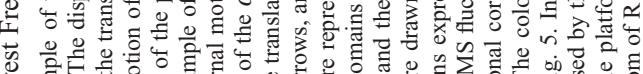

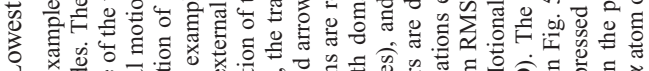

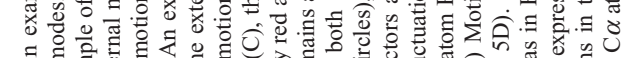

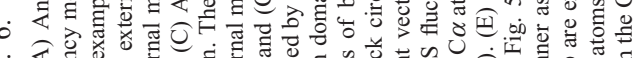

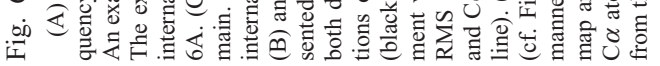
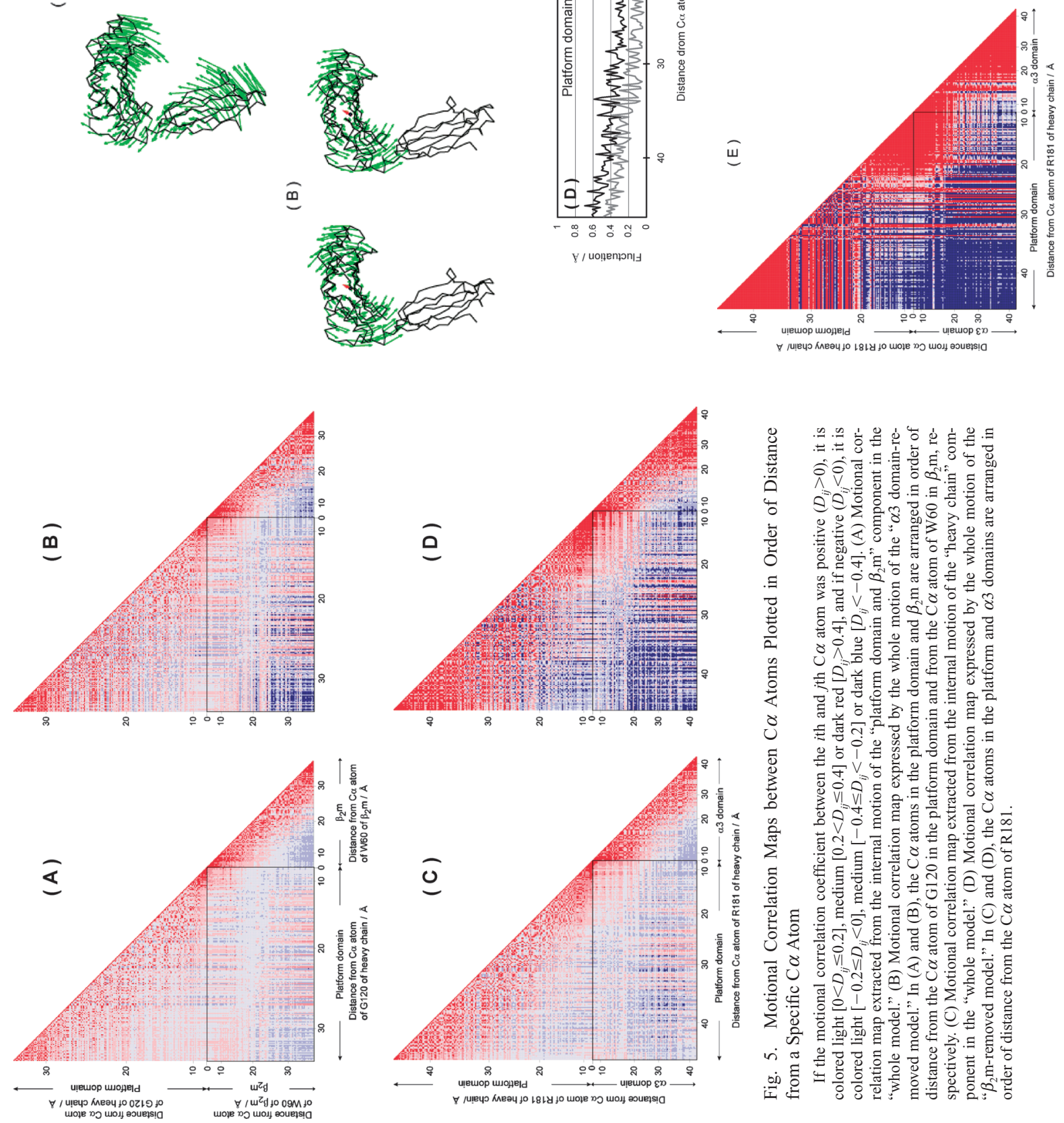
Table 2. The Lowest, Second-Lowest and Third-Lowest Frequency Modes Obtained from Normal Mode Analysis

\begin{tabular}{|c|c|c|c|c|c|c|c|}
\hline & \multirow{2}{*}{ Mode no. } & \multicolumn{6}{|c|}{ Optimized structure no. } \\
\hline & & 1 & 2 & 3 & 4 & 5 & 6 \\
\hline \multirow[t]{3}{*}{ "Whole model" } & 1672 & 6.05 & 5.02 & 5.98 & 6.08 & 6.31 & 4.78 \\
\hline & 1673 & 4.64 & 4.4 & 4.59 & 4.57 & 4.95 & 4.48 \\
\hline & 1674 & 3.33 & $2.94^{a)}$ & 3.24 & 3.39 & 3.34 & 3.22 \\
\hline \multirow[t]{3}{*}{ " $\alpha 3$ domain-removed model" } & 1287 & 5.05 & 5.11 & 5.58 & 5.56 & 5.7 & 5.02 \\
\hline & 1288 & 3.61 & 4.21 & 4.21 & 4.17 & 4.26 & 3.71 \\
\hline & 1289 & 3.24 & 3.29 & 3.31 & 3.21 & 3.39 & 3.22 \\
\hline \multirow[t]{3}{*}{ " $\beta_{2}$ m-removed model" } & 1222 & 3.04 & 2.88 & 2.97 & 3.01 & 2.96 & 2.95 \\
\hline & 1223 & 2.51 & 2.22 & 2.35 & 2.47 & 2.33 & 2.23 \\
\hline & 1224 & 1.97 & 1.8 & 1.71 & 1.75 & 1.72 & 1.74 \\
\hline
\end{tabular}

a) Low-frequency modes $<3 \mathrm{~cm}^{-1}$ are given as bold figures.

other hand, when the motional correlation map expressed by the whole motion of the " $\beta_{2}$ m-removed model" (Fig. 5D) was compared with that extracted from the "heavy chain" component of the "whole model" (Fig. 5C), the " $\beta_{2}$ m-removed model" showed definite negative correlations (motional correlation coefficient $<-0.4$ ) in most of between the platform and the $\alpha 3$ domains (in the black frame).

Motions of Low Frequency Modes In this study, we investigated low frequency modes because they generally influence the collective motion of large molecules such as class I MHC molecules more than high-frequency modes do. ${ }^{38)} \mathrm{Al}-$ most all of the lowest frequency modes obtained for the simulations of the "whole model" and the " $\alpha 3$ domain-removed model" were $>3 \mathrm{~cm}^{-1}$ (Table 2). On the other hand, almost all of the lowest, second-lowest and third-lowest frequency modes obtained for the simulations of the " $\beta_{2}$ m-removed model" were $<3 \mathrm{~cm}^{-1}$, and all of the lowest frequency modes were $<2 \mathrm{~cm}^{-1}$ (Table 2). The lowest frequency modes of the " $\beta_{2}$ m-removed model" expressed clear interdomain motions, as if each of the platform and $\alpha 3$ domains moved like a rigid body (an example is shown in Fig. 6A), and such clear interdomain motions were not observed for the lowest frequency modes of the other models. Generally, when the internal motion of a domain is removed from the whole motion of molecules, the external motion (translational and rotational motions) of the domain can be extracted. The extraction of the external motion from the whole motion expressed in Fig. $6 \mathrm{~A}$ revealed the translational motions of both domains in exactly opposite directions to each other $\left(\theta=179.4^{\circ}\right)$ and the clear rotational motions of both domains (Figs. $6 \mathrm{~B}$ and $\mathrm{C}$ ).

The lowest frequency modes of the " $\beta_{2}$ m-removed model" expressed similar fluctuation patterns to those expressed in all of the low frequency modes $\left(<50 \mathrm{~cm}^{-1}\right)$, that is, the fluctuations increased with distance from the linker position (Fig. 6D). The motional correlation map of the " $\beta_{2}$ m-removed model" expressed in only the lowest frequency modes showed clear negative correlations in most of between the platform and $\alpha 3$ domains except for near the linker position (Fig. 6E). On the other hand, the other low frequency modes $\left(<50 \mathrm{~cm}^{-1}\right)$ of the " $\beta_{2}$ m-removed model," except for the lowest frequency modes, showed similar fluctuation patterns and motional correlation profiles to all of the low frequency modes $\left(<50 \mathrm{~cm}^{-1}\right)$ of the "whole model" (data is not shown).

\section{Discussion}

The " $\beta_{2}$ m-removed model" showed larger fluctuations and clearer negative correlations in interdomain than the " $\alpha 3$ domain-removed model." This result indicates that the " $\beta_{2} \mathrm{~m}$-removed model" is more flexible in interdomain conformation than the " $\alpha 3$ domain-removed model." The lowest frequency modes $\left(1.71-1.97 \mathrm{~cm}^{-1}\right.$, see Table 2$)$ observed for the simulation of the " $\beta_{2}$ m-removed model" corresponded to a time scale of $16.9-19.5 \mathrm{ps}$ per period. The lowest frequency modes were peculiar to the " $\beta_{2}$ m-removed model" because such low frequencies and clear interdomain motions as if each domain moved like a rigid body were not observed for the simulations of other models. The flexibility of the " $\beta_{2} \mathrm{~m}$ removed model" in interdomain conformation versus the " $\alpha 3$ domain-removed model," therefore, may be due to the lowest frequency modes.

The above results are worthy of note when considering the interaction among the three domains. It is well known that endogenous $\beta_{2} \mathrm{~m}$ in class I MHC molecules is easily released for exchange with exogenous $\beta_{2} \mathrm{~m}$ on the cell surface. ${ }^{2-7)}$ The behavior of the " $\beta_{2}$ m-removed model" suggested the flexibility of the interdomain conformation after releasing $\beta_{2} \mathrm{~m}$ from the complete molecules. For example, the relationship between the platform and $\alpha 3$ domains may dramatically change as if both domains are linearly arranged, and each domain may behave without interaction with each other. The structural stability of each domain in the " $\beta_{2}$ m-removed model" could not be discussed from this simulation. However, Wan et al. have compared the behavior between a whole model composed of all domains and a partial model composed of only the platform domain, and have reported that the peptide is less tightly bound in the partial model than in the whole model. ${ }^{17)}$ Furthermore, it is experimentally and computationally well known that the structure of the platform domain in the peptide-free molecules is thermally more unstable than that in the peptide-filled molecules. ${ }^{15,40)}$ On the other hand, the behavior of the " $\alpha 3$ domain-removed model" suggests that the interdomain conformation is fixed regardless of the $\alpha 3$ domain, and that molecules deficient in the $\alpha 3$ domain have a more stable conformation than those deficient in $\beta_{2} \mathrm{~m}$. Indeed, while the molecules deficient in $\beta_{2} \mathrm{~m}$ have never been crystallized, those deficient in the $\alpha 3$ domain has been crystallized and their structure is similar to the corresponding part of the intact structure including the $\alpha 3$ domain. ${ }^{8)}$

The behavior of the " $\beta_{2}$ m-removed model" and " $\alpha 3$ domain-removed model" observed by normal mode analysis suggests that $\beta_{2} \mathrm{~m}$ contribute to maintaining the interdomain 
conformation of class I MHC molecules more than the $\alpha 3$ domain does, and may offer convincing evidence to support the notion that the $\alpha 3$ domain and $\beta_{2} \mathrm{~m}$ do not exert an equal influence on the structural stability of class I MHC molecules.

\section{References}

1) Madden D. R., Garboczi D. N., Wiley D. C., Cell, 75, 693-708 (1993).

2) Rock K. L., Gamble S., Rothstein L., Benacerraf B., Proc. Natl. Acad. Sci. U.S.A., 88, 301-304 (1991).

3) Kozlowski S., Takeshita T., Boehncke W. H., Takahashi H., Boyd L. F., Germain R. N., Berzofsky J. A., Margulies D. H., Nature (London), 349, 74-77 (1991).

4) Vitiello A., Potter T. A., Sherman L. A., Science, 250, 1423-1426 (1990).

5) Kane K. P., Sherman L. A., Mescher M. F., Eur. J. Immunol., 21, 2289-2292 (1991).

6) Rock K. L., Rothstein L. E., Gamble S. R., Benacerraf B., Proc. Natl. Acad. Sci. U.S.A., 87, 7517-7521 (1990).

7) Cook J. R., Myers N. B., Hansen T. H., Immunology, 157, 2256-2261 (1996).

8) Collins E. J., Garboczi D. N., Karpusas M. N., Wiley D. C., Proc. Natl. Acad. Sci. U.S.A., 92, 1218-1221 (1995).

9) Rognan D., Reddehase M. J., Koszinowski U. H., Folkers G., Proteins, 13, 70-85 (1992).

10) Meng W. S., von Grafenstein H., Haworth I. S., Int. Immunol., 9, 1339-1346 (1997).

11) Toh H., Kamikawaji N., Tana T., Muta S., Sasazuki T., Kuhara S., Protein Eng., 13, 423- 429 (2000).

12) Meng W. S., von Grafenstein H., Haworth I. S., Int. Immunol., 12, 949-957 (2000)

13) Mandal T. K., Mukhopadhyay C., Biopolymers, 59, 11-23 (2001).

14) Michielin O., Karplus M., J. Mol. Biol., 324, 547-569 (2002).

15) Zacharias M., Springer S., Biophys. J., 87, 2203-2214 (2004).

16) Sieker F., Springer S., Zacharias M., Protein Sci., 16, 299-308 (2007).

17) Wan S., Coveney P. V., Flower D. R., J. Comput. Chem., 25, 1803-
1813 (2004).

18) Levitt M., Sander C., Stern P. S., J. Mol. Biol., 181, $423-447$ (1985).

19) Brooks B., Karplus M., Proc. Natl. Acad. Sci. U.S.A., 80, 6571-6575 (1983).

20) Nishikawa T., Go N., Proteins, 2, 308-329 (1987)

21) Wako H., J. Protein Chem., 8, 589-607 (1989).

22) Miller D. W., Agard D. A., J. Mol. Biol., 286, 267-278 (1999).

23) Ishida H., Jochi Y., Kidera A., Proteins, 32, 324-333 (1998).

24) Jääskeläinen S., Verma C. S., Hubbard R. E., Linko P., Caves L. S., Protein Sci., 7, 1359-1367 (1998)

25) Hayward S., Kitao A., Berendsen H. J., Proteins, 27, 425-437 (1997).

26) Kamiya K., Sugawara Y., Umeyama H., J. Comput. Chem., 24, 826841 (2003).

27) Weiner S. J., Kollman P. A., Case D. A., Singh U. C., Ghio C., Alagona G., Profeta S. J., Weiner P., J. Am. Chem. Soc., 106, 765-784 (1984).

28) Weiner S. J., Kollman P. A., Nguyen D. T., Case D. A., J. Comput Chem., 7, 230-252 (1986)

29) Loncharich R. J., Brooks B. R., Proteins, 6, 32-45 (1989).

30) Nojima H., Takeda-Shitaka M., Kurihara Y., Adachi M., Yoneda S., Kamiya K., Umeyama H., Chem. Pharm. Bull., 50, 1209-1214 (2002).

31) Nojima H., Takeda-Shitaka M., Kurihara Y., Kamiya K., Umeyama H., Chem. Pharm. Bull., 51, 923-928 (2003).

32) Adachi M., Kurihara Y., Nojima H., Takeda-Shitaka M., Kamiya K., Umeyama H., Protein Sci., 12, 2125-2131 (2003).

33) Kurihara Y., Watanabe T., Nojima H., Takeda-Shitaka M., Kamiya K., Umeyama H., Chem. Pharm. Bull., 51, 754-758 (2003).

34) Soejima K., Kurihara Y., Kamiya K., Umeyama H., FEBS Lett., 463, 19-23 (1999).

35) Sumikawa H., Suzuki E., Fukuhara K., Nakajima Y., Kamiya K., Umeyama H., Chem. Pharm. Bull., 46, 1069-1077 (1998).

36) Takeda-Shitaka M., Kamiya K., Miyata T., Ohkura N., Madoiwa S., Sakata Y., Umeyama H., Chem. Pharm. Bull., 47, 322-328 (1999).

37) Higo J., Umeyama H., Protien Eng., 186, 477-490 (1997).

38) Go N., Noguti T., Nishikawa T., Proc. Natl. Acad. Sci. U.S.A., 80, 3696-3700 (1983).

39) Eckart C., Phys. Rev., 47, 552-558 (1935).

40) Bouvier M., Wiley D. C., Nat. Struct. Biol., 5, 377-384 (1998). 